\title{
The generalized second law of thermodynamics with Barrow entropy
}

\author{
Emmanuel N. Saridakis ${ }^{1,2,3, a}{ }_{\mathbb{D}}$, Spyros Basilakos $^{4, \mathrm{~b}}$ \\ ${ }^{1}$ National Observatory of Athens, Lofos Nymfon, 11852 Athens, Greece \\ ${ }^{2}$ CAS Key Laboratory for Researches in Galaxies and Cosmology, Department of Astronomy, University of Science and Technology of China, \\ Hefei 230026, Anhui, People's Republic of China \\ ${ }^{3}$ School of Astronomy, School of Physical Sciences, University of Science and Technology of China, Hefei 230026, People's Republic of China \\ ${ }^{4}$ Academy of Athens, Research Center for Astronomy and Applied Mathematics, Soranou Efesiou 4, 11527 Athens, Greece
}

Received: 14 December 2020 / Accepted: 7 July 2021 / Published online: 22 July 2021

(C) The Author(s) 2021

\begin{abstract}
We investigate the validity of the generalized second law of thermodynamics, applying Barrow entropy for the horizon entropy. The former arises from the fact that the black-hole surface may be deformed due to quantumgravitational effects, quantified by a new exponent $\Delta$. We calculate the entropy time-variation in a universe filled with the matter and dark energy fluids, as well as the corresponding quantity for the apparent horizon. We show that although in the case $\Delta=0$, which corresponds to usual entropy, the sum of the entropy enclosed by the apparent horizon plus the entropy of the horizon itself is always a non-decreasing function of time and thus the generalized second law of thermodynamics is valid, in the case of Barrow entropy this is not true anymore, and the generalized second law of thermodynamics may be violated, depending on the universe evolution. Hence, in order not to have violation, the deformation from standard Bekenstein-Hawking expression should be small as expected.
\end{abstract}

\section{Introduction}

There is a well known analogy between black-hole physics and thermodynamics. In particular, one can attribute to a black hole a specific temperature and entropy, which depend on the black-hole horizon [1]. Inspired by this, an extension of this analogy was proposed, namely the conjecture of the "thermodynamics of spacetime", according to which one can apply thermodynamics in the horizon of the universe. In particular, thermodynamical laws are applied on the horizon itself, considered as a system separated not by a diathermic wall but by a causality barrier (i.e. the "system" is composed

\footnotetext{
a e-mail: msaridak@phys.uoa.gr (corresponding author)

b e-mail: svasil@academyofathens.gr
}

by the degrees of freedom beyond the horizon) [2-4]. Thus, these laws are interpreted in terms of area of local Rindler horizons and energy flux [2], and heat is incorporated as energy that flows through the causal horizon. Concerning the relations for temperature and entropy of the horizon, these are given by the corresponding relations of black hole thermodynamics, but with the universe horizon in place of the black-hole horizon.

Applying the first law of thermodynamics on the apparent horizon one can extract the Friedmann equations, and reversely one can express the Friedmann equations as the first law [5-7]. This procedure proves to be applicable both in general relativity as well as in a variety of modified gravity theories, despite the fact that in the latter theories the entropy relation is in general modified [8-17]. Concerning the second law, which in black-hole physics had been extended to the "generalized second law of thermodynamics", namely that the usual entropy plus the blackhole horizon entropy is a non-decreasing function of time $[18,19]$, one can also apply it to the universe horizon and claim that the total entropy of the interior of the universe plus the entropy of its horizon should be a non-decreasing function of time [20]. Note that this statement is proven to be always valid for general relativity in FriedmannRobertson-Walker geometry, however it is not always the case in modified theories of gravity and hence one can apply it in order to extract constraints on them [14,2132]. Since the generalized second law of thermodynamics is fundamental in physics, its (conditional or complete) violation acts as a strong argument against the underlying theory.

Recently, Barrow [33] considered the case that quantumgravitational effects might bring about intricate, fractal structure on the black hole surface and thus changing its actual 
horizon area. This in turn led to a new black hole entropy relation, i.e.

$S_{B}=\left(\frac{A}{A_{0}}\right)^{1+\Delta / 2}$,

where $A$ is the usual horizon area and $A_{0}$ the Planck area. Note that the above deformed entropy differs from the usual "quantum-corrected" one with logarithmic corrections [34,35], however it resembles Tsallis nonextensive entropy [36-38], nevertheless the involved physical principles and foundations are radically different. The quantumgravitational deformation is quantified by the new exponent $\Delta$. The value $\Delta=0$ corresponds to the simplest horizon structure, and in this case we obtain the standard BekensteinHawking entropy, while $\Delta=1$ corresponds to maximal deformation.

In the present work we are interested in examining the validity of the generalized second law of thermodynamics, but applying the Barrow entropy on the horizon instead of the standard Bekenstein-Hawking one.

\section{Generalized second law of thermodynamics}

In this section we will apply the generalized second law of thermodynamics in the universe using Barrow entropy. We consider a spatially homogeneous and isotropic FriedmannRobertson-Walker metric

$d s^{2}=-d t^{2}+a^{2}(t) \delta_{i j} d x^{i} d x^{j}$.

Additionally, we consider that the universe is filled with matter and dark energy perfect fluids, with energy density and pressure $\rho_{D E}, \rho_{m}$ and $p_{D E}, p_{m}$ respectively (as usual we do not take into account the black-hole formation inside the universe and its effect on entropy). Thus, the two Friedmann equations are

$$
\begin{aligned}
& H^{2}=\frac{8 \pi G}{3}\left(\rho_{m}+\rho_{D E}\right) \\
& \dot{H}=-4 \pi G\left(\rho_{m}+p_{m}+\rho_{D E}+p_{D E}\right),
\end{aligned}
$$

with $H=\dot{a} / a$ the Hubble parameter and where a dot denotes the derivative with respect to $t$. Note that the aforementioned framework holds in general, independently of the specific dark energy description. Finally, the conservation of the total energy-momentum tensor gives:

$\dot{\rho}_{D E}+3 H\left(1+w_{D E}\right) \rho_{D E}+\dot{\rho}_{m}+3 H\left(1+w_{m}\right) \rho_{m}=0$,

where $w_{i}=p_{i} / \rho_{i}$ denotes the equation-of-state parameter of the corresponding sector, which throughout this work are considered general and not constant. We mention that the above relation holds both in the usual case, as well as in the case where the two sectors are allowed to mutually interact.

Concerning the universe horizon, that will be the boundary of the thermodynamical system, although in the literature there have been discussed various choices, there are many arguments that the appropriate one should be the apparent horizon, which is a marginally trapped surface with vanishing expansion given by [39-41]:

$\tilde{r}_{A}=\frac{1}{\sqrt{H^{2}+\frac{k}{a^{2}}}}$,

where $k$ quantifies the spatial curvature which is set to zero in the present work. Hence, the first Friedmann equation (3) becomes

$\frac{1}{\tilde{r}_{A}^{2}}=\frac{8 \pi G}{3}\left(\rho_{D E}+\rho_{m}\right)$

We are going to investigate whether the sum of the entropy enclosed by the apparent horizon plus the entropy of the apparent horizon itself, is not a decreasing function of time. We will start by calculating the former and then the latter.

In general, the apparent horizon $\tilde{r}_{A}$ is time-dependent. Hence, a change $d \tilde{r}_{A}$ in time interval $d t$ will bring about a volume-change $d V$, while the energy and entropy of the universe fluids will change by $d E$ and $d S$ respectively. Now, the first law of thermodynamics applied in the universe is written as $T d S=d E+P d V$, and therefore the dark-energy and dark-matter entropies read [42]:

$$
\begin{aligned}
d S_{D E} & =\frac{1}{T}\left(P_{D E} d V+d E_{D E}\right) \\
d S_{m} & =\frac{1}{T}\left(P_{m} d V+d E_{m}\right) .
\end{aligned}
$$

Since the universe volume, bounded by the apparent horizon, is $V=4 \pi \tilde{r}_{A}^{3} / 3$, we obtain $d V=4 \pi \tilde{r}_{A}^{2} d \tilde{r}_{A}$. Additionally, concerning the temperature of the universe fluids, we assume it to be the same due to the establishment of equilibrium. Dividing (8), (9) by $d t$ we acquire

$$
\begin{aligned}
\dot{S}_{D E} & =\frac{1}{T}\left(P_{D E} 4 \pi \tilde{r}_{A}^{2} \dot{\tilde{r}}_{A}+\dot{E}_{D E}\right) \\
\dot{S}_{m} & =\frac{1}{T}\left(P_{m} 4 \pi \tilde{r}_{A}^{2} \dot{\tilde{r}}_{A}+\dot{E}_{m}\right)
\end{aligned}
$$

where

$\dot{\tilde{r}}_{A}=-\dot{H} \tilde{r}_{A}^{2}$,

as it easily arises differentiating (6). 
In order to connect the thermodynamical quantities $E_{i}$ and $P_{i}$, with the cosmological $\rho_{i}$ and $p_{i}$ ones, we straightforwardly use

$$
\begin{aligned}
E_{D E} & =\frac{4 \pi}{3} \tilde{r}_{A}^{3} \rho_{D E} \\
E_{m} & =\frac{4 \pi}{3} \tilde{r}_{A}^{3} \rho_{m} .
\end{aligned}
$$

Inserting the time-derivatives of (13), (14), into (10), (11) and using (5), we obtain:

$$
\begin{aligned}
\dot{S}_{D E} & =\frac{1}{T}\left(1+w_{D E}\right) \rho_{D E} 4 \pi \tilde{r}_{A}^{2}\left(\dot{\tilde{r}}_{A}-H \tilde{r}_{A}\right) \\
\dot{S}_{m} & =\frac{1}{T}\left(1+w_{m}\right) \rho_{m} 4 \pi \tilde{r}_{A}^{2}\left(\dot{\tilde{r}}_{A}-H \tilde{r}_{A}\right) .
\end{aligned}
$$

These expressions provide the entropy time-variation for the universe interior.

We now proceed to the calculation of the entropy timevariation for the universe horizon. As we mentioned in the introduction, according to the "gravity-thermodynamics" conjecture the temperature and entropy of the horizon will be given by the corresponding quantities of black-hole thermodynamics, but with the apparent horizon in place of the black-hole one. The horizon temperature will thus be simply $[2,6]$

$$
T_{h}=\frac{1}{2 \pi \tilde{r}_{A}} .
$$

Concerning the horizon entropy, the standard choice is to use the Bekenstein-Hawking entropy [2,6] $S_{h}=4 \pi \tilde{r}_{A}^{2} /(4 G)$. However, in the present work we will instead apply the Barrow black-hole entropy (1), with the standard horizon area being $A=4 \pi \tilde{r}_{A}^{2}$. Therefore, we obtain

$S_{h}=\gamma \tilde{r}_{A}^{\Delta+2}$

with $\gamma \equiv\left(4 \pi / A_{0}\right)^{1+\Delta / 2}$. Finally, a crucial assumption in the "gravity-thermodynamics" conjecture is that after equilibrium establishes the universe fluids acquire the same temperature with the horizon one, which is constant or slowlyvarying, otherwise the energy flow would deform this geometry [43] (in order to avoid applying non-equilibrium thermodynamics the assumption of equilibrium is widely used [4-7, 14,43-46], see also [47]). Therefore, we can equate $T_{h}$ in (17) with $T$ in (15), (16), and the temperature is only slowly evolving according to (17) due to the slow change of the apparent horizon. Lastly, differentiating (18) we obtain

$$
\dot{S}_{h}=(\Delta+2) \gamma \tilde{r}_{A}^{\Delta+1} \dot{\tilde{r}}_{A} .
$$

We can now calculate the total entropy time-variation. Adding relations (15), (16) and (19), and replacing $T$ through (17), we find:

$$
\begin{aligned}
\dot{S}_{t o t} & \equiv \dot{S}_{D E}+\dot{S}_{m}+\dot{S}_{h} \\
& =8 \pi^{2} \tilde{r}_{A}^{3}\left(\dot{\tilde{r}}_{A}-H \tilde{r}_{A}\right)\left[\left(1+w_{D E}\right) \rho_{D E}+\left(1+w_{m}\right) \rho_{m}\right] \\
& +(\Delta+2) \gamma \tilde{r}_{A}^{\Delta+1} \dot{\tilde{r}}_{A} .
\end{aligned}
$$

Hence, substituting $\dot{\tilde{r}}_{A}$ from (12), knowing that $\tilde{r}_{A}=H^{-1}$, and using the two Friedmann equations (3), (4), we easily find

$\dot{S}_{t o t}=\frac{2 \pi}{G} H^{-5} \dot{H}\left\{\dot{H}+H^{2}\left[1-\frac{\gamma G}{2 \pi}(\Delta+2) H^{-\Delta}\right]\right\}$.

Let us now examine the sign of (21). In the case where the Barrow exponent takes its standard value $\Delta=0$, i.e. in the case of usual black-hole thermodynamics, we have

$\left.\dot{S}_{t o t}\right|_{\Delta=0}=\frac{2 \pi}{G} H^{-5} \dot{H}^{2} \geq 0$,

since in this case $\gamma \equiv\left(4 \pi / A_{0}\right)=\pi / G$ (in units where $\left.\hbar=k_{B}=c=1\right)$. As expected, $\left.\dot{S}_{t o t}\right|_{\Delta=0} \geq 0$ and thus the generalized second law of thermodynamic is always valid in a universe filled with matter and dark energy sectors and governed by general relativity (note that the limiting result $\left.\dot{S}_{t o t}\right|_{\Delta=0}=0$ is obtained for the de Sitter universe).

However, interestingly enough, in the case where the Barrow exponent is non-zero, i.e. the quantum-gravitational effects on the entropy switch on, the total entropy is not necessarily a non-decreasing function of time, and hence the generalized second law can be violated. The reason is the following.

In the case of standard Bekenstein-Hawking universe thermodynamics, $\dot{S}_{h}$ has always the necessary value in order to bring $\dot{S}_{\text {tot }}$ to non-negative values. In particular, for $\dot{H}<0$, i.e. in the case where the universe fluids satisfy the null energy condition in total, we have $\dot{S}_{h}>0$ and thus it leads to $\dot{S}_{t o t} \geq 0$ even if the universe fluids have $\dot{S}_{D E}+\dot{S}_{m}<0$, while in the case $\dot{H}>0$, i.e for the violation of the total null energy condition, where $\dot{S}_{h}<0$ we have $\dot{S}_{D E}+\dot{S}_{m}>0$ always in a sufficient amount to make $\dot{S}_{\text {tot }} \geq 0$. However, in the case where Barrow entropy is used the horizon contributes with a changed $\dot{S}_{h}$, which does not satisfy the aforementioned conditions anymore (in the case where it is positive it is not always sufficiently positive to counterbalance cases where $\dot{S}_{D E}+\dot{S}_{m}<0$, while in the case where it is negative it is not always sufficiently close to zero in order to be counterbalanced by the $\dot{S}_{D E}+\dot{S}_{m}>0$ ). Therefore, the use of the deformed, Barrow entropy, leads to the conditional violation 


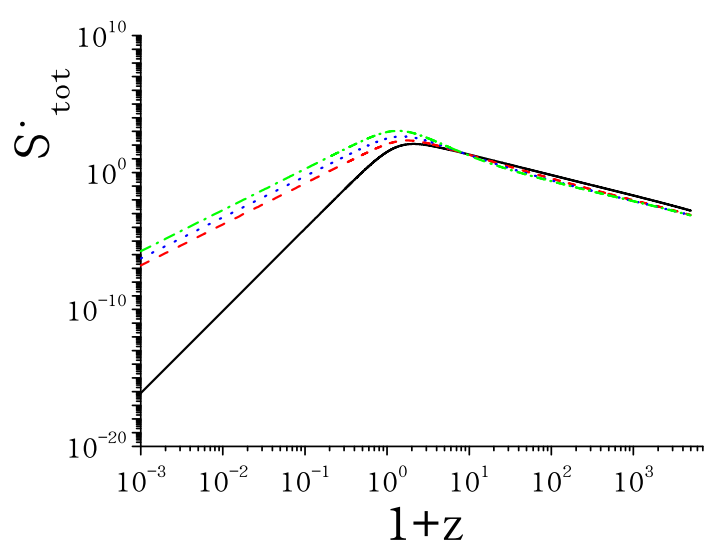

Fig. 1 The evolution of the entropy time-variation as a function of the redshift $z$ given by (23), in the case of $\Lambda$ CDM background evolution (24), for various values of the Barrow exponent $\Delta: \Delta=0$ (black-solid), $\Delta=0.3$ (red-dashed), $\Delta=0.6$ (blue-dotted), $\Delta=1$ (green-dashed-dotted). We have imposed $\Omega_{m 0}=0.30$ and $\Omega_{r 0}=10^{-4}$, and we have used units where $\hbar=k_{B}=c=1=H_{0}=1$

of the generalized second law of thermodynamics, depending on the universe evolution.

In order to see the above qualitative behavior more transparently we proceed to a quantitative investigation, by exploring $\dot{S}_{t o t}$ for different forms of Hubble function evolution. For convenience we use the redshift $1+z=a_{0} / a$ (with $a_{0}=1$ the present scale factor) as the independent variable, and thus relation (21) becomes

$$
\begin{aligned}
\dot{S}_{\text {tot }}(z)= & \frac{2 \pi}{G} H(z)^{-3} H^{\prime}(z)(1+z)\left\{H^{\prime}(z)(1+z)\right. \\
& \left.-H(z)\left[1-\frac{\gamma G}{2 \pi}(\Delta+2) H(z)^{-\Delta}\right]\right\},
\end{aligned}
$$

where primes denote derivatives with respect to $z$ and we have used the relation $\dot{f}=-(1+z) H f^{\prime}$.

As a first example we consider the case where the Hubble function evolves as in $\Lambda \mathrm{CDM}$ cosmology, namely as

$$
H_{\Lambda \mathrm{CDM}}(z)=H_{0} \sqrt{\Omega_{m 0}(1+z)^{3}+\Omega_{r 0}(1+z)^{4}+\Omega_{\Lambda 0}},
$$

where $H_{0}$ is the current Hubble parameter, $\Omega_{i 0}$ is the value of the density parameter $\Omega_{i}=8 \pi G \rho_{i} /\left(3 H^{2}\right)$ at present, $\Omega_{\Lambda 0}=1-\Omega_{m 0}-\Omega_{r 0}$, and with the subscripts " $m$ " and " $\mathrm{r}$ " denoting the matter and radiation sectors respectively. We insert (24) into (23) and in Fig. 1 we depict the evolution of $\dot{S}_{t o t}(z)$ for various values of the Barrow exponent $\Delta$. As we observe, $\dot{S}_{t o t}$ is always positive, throughout cosmological evolution, and for all values of $\Delta$. This is an advantage for Barrow entropy, since it implies that for $\Lambda \mathrm{CDM}$ background the generalized second law of thermodynamics is always valid.
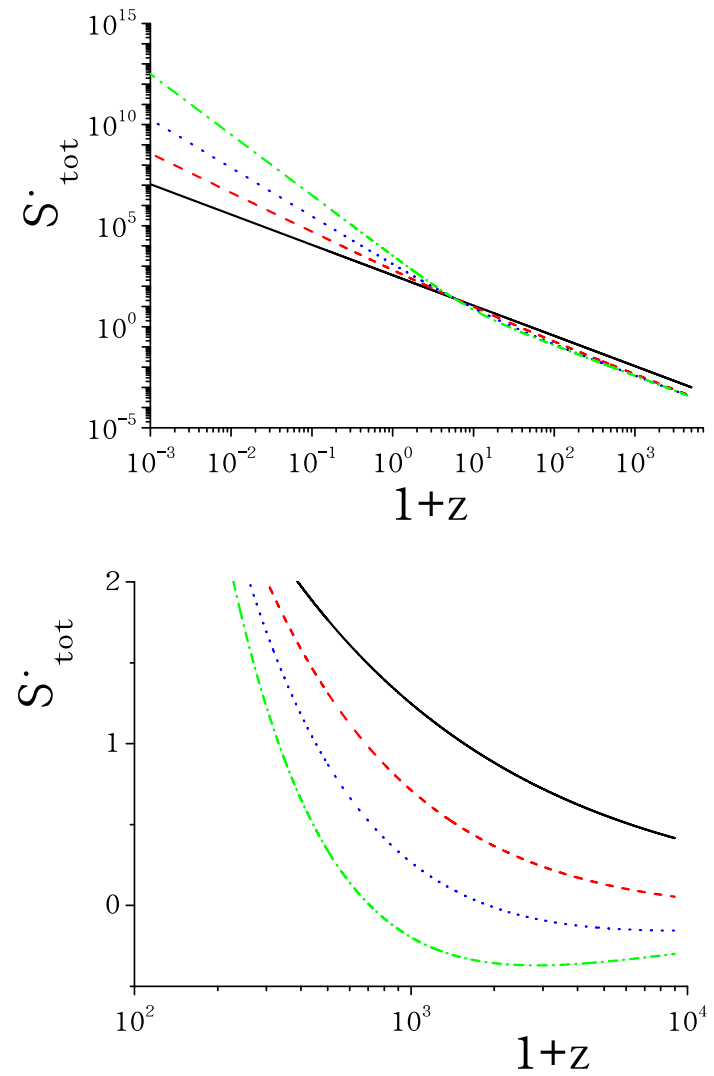

Fig. 2 The evolution of the entropy time-variation as a function of the redshift $z$ given by (23), in the case of power-law background evolution (25), for various values of the Barrow exponent $\Delta$. Upper panel: $n=$ $2 / 3$ and $\Delta=0$ (black-solid), $\Delta=0.3$ (red-dashed), $\Delta=0.6$ (blue-dotted), $\Delta=1$ (green-dashed-dotted). Lower panel (mind the difference in the axes): $n=2$ and $\Delta=0$ (black-solid), $\Delta=$ 0.3 (red-dashed), $\Delta=0.6$ (blue-dotted), $\Delta=1$ (green-dasheddotted). We have imposed $\Omega_{m 0}=0.30$ and $\Omega_{r 0}=10^{-4}$, and we have used units where $\hbar=k_{B}=c=1=H_{0}=1$

Let us proceed by investigating a general power-law cosmological evolution of the form $a(t)=a_{1} t^{n}$, which implies that

$H_{\text {power }}(z)=H_{0}(1+z)^{1 / n}$

with $H_{0}=n a_{1}^{1 / n}$, which is a well studied scenario in cosmology. Inserting (25) into (23) we find

$$
\begin{aligned}
\dot{S}_{\text {tot }}(z)= & \frac{\left[H_{0}(z+1)^{1 / n}\right]^{-1-\Delta}}{G n^{2}}\{G n \gamma(2+\Delta) \\
& \left.+2(1-n) \pi\left[H_{0}(z+1)^{1 / n}\right]^{\Delta}\right\},
\end{aligned}
$$

and in Fig. 2 we depict the evolution of $\dot{S}_{t o t}(z)$ for various values of the Barrow exponent $\Delta$, considering $n=2 / 3$ (upper panel) and $n=2$ (lower panel).

As we can see, in the case where $n<1$ the entropy timevariation is always positive for all $\Delta$ values, and thus the generalized second law of thermodynamics is always valid 
(this can be immediately seen from the analytical expression (26) too). However, in the case $n>1$ we observe that for suitably large $\Delta$ values $\dot{S}_{\text {tot }}$ can become negative in the past (this can be implied from (26) too), and thus the generalized second law is violated. Hence, these parameter regions should be avoided.

We close this section by mentioning that although Barrow entropy accounts for quantum-gravitational phenomena, since the gravity-thermodynamics conjecture incorporates holography, which in turn dually connects the very small with the very large, one in principle may have the reflection of these phenomena on the classical universe horizon itself (this is also the case of the usual logarithmic-corrected entropy due to quantum effects, which is widely studied in the universethermodynamics framework as well [34,35,48,49]). Nevertheless, what is the value of $\Delta$ in the real world is a different thing, and actually one expects it to be close to the standard value $\Delta=0$. Indeed, recent cosmological observational constraints on $\Delta$ reveal that the value zero is indeed inside the $1 \sigma$ region [50].

\section{Conclusions}

In this work we investigated the validity of the generalized second law of thermodynamics, but using for the horizon entropy the Barrow one. Specifically, Barrow entropy arises from the fact that the black-hole surface may be deformed due to quantum-gravitational effects, and its deviation from Bekenstein-Hawking one is quantified by a new exponent $\Delta$.

We calculated the entropy time-variation in a universe filled with the matter and dark energy fluids, as well as the corresponding quantity for the apparent horizon. As we showed, although in the case $\Delta=0$, which corresponds to usual entropy, the sum of the entropy enclosed by the apparent horizon plus the entropy of the horizon itself is always a nondecreasing function of time and thus the generalized second law of thermodynamics is valid, in the case where quantumgravitational corrections switch on this is not true anymore. Hence, the generalized second law of thermodynamics may be conditionally violated, depending on the universe evolution. In particular, in the case of $\Lambda \mathrm{CDM}$ background cosmological evolution the entropy time-variation is always positive and thus the generalized second law of thermodynamics is always valid. However, in the case of a general power-law cosmological evolution we found that for power-law exponents larger than one and for suitably large $\Delta$ values the entropy time-variation can be negative in the past, leading to the violation of the generalized second law. Hence, the involved parameter regions should be avoided, and therefore if Barrow entropy is the case in nature then its allowed region would be constrained in a narrow window close to the stan- dard entropy. This reveals the usefulness of examining the generalized second law of thermodynamics.

It would be interesting to investigate whether the above result remains valid in the case of various gravitational modifications instead of general relativity, and whether the known violations of the generalized second law in modified gravity may be eliminated using Barrow entropy. These studies lie beyond the scope of the present work and are left for future projects.

Data Availability Statement This manuscript has no associated data or the data will not be deposited. [Authors' comment: This is a theoretical work, and hence no data have been used.]

Open Access This article is licensed under a Creative Commons Attribution 4.0 International License, which permits use, sharing, adaptation, distribution and reproduction in any medium or format, as long as you give appropriate credit to the original author(s) and the source, provide a link to the Creative Commons licence, and indicate if changes were made. The images or other third party material in this article are included in the article's Creative Commons licence, unless indicated otherwise in a credit line to the material. If material is not included in the article's Creative Commons licence and your intended use is not permitted by statutory regulation or exceeds the permitted use, you will need to obtain permission directly from the copyright holder. To view a copy of this licence, visit http://creativecomm ons.org/licenses/by/4.0/.

Funded by $\mathrm{SCOAP}^{3}$.

\section{References}

1. G.W. Gibbons, S.W. Hawking, Phys. Rev. D 15, 2738 (1977) https://doi.org/10.1103/PhysRevD.15.2738

2. T. Jacobson, Phys. Rev. Lett. 75, 1260 (1995). https://doi.org/10. 1103/PhysRevLett.75.1260. arXiv:gr-qc/9504004

3. T. Padmanabhan, Phys. Rep. 406, 49 (2005). https://doi.org/10. 1016/j.physrep.2004.10.003. arXiv:gr-qc/0311036

4. T. Padmanabhan, Rep. Prog. Phys. 73, 046901 (2010). https://doi. org/10.1088/0034-4885/73/4/046901. arXiv:0911.5004 [gr-qc]

5. A.V. Frolov, L. Kofman, JCAP 0305, 009 (2003). https://doi.org/ 10.1088/1475-7516/2003/05/009. arXiv:hep-th/0212327

6. R.G. Cai, S.P. Kim, JHEP 0502, 050 (2005). https://doi.org/10. 1007/JHEP06(2014)093. arXiv:hep-th/0501055

7. M. Akbar, R.G. Cai, Phys. Rev. D 75, 084003 (2007). https://doi. org/10.1103/PhysRevD.75.084003. arXiv:hep-th/0609128

8. A. Paranjape, S. Sarkar, T. Padmanabhan, Phys. Rev. D 74, 104015 (2006). https://doi.org/10.1103/PhysRevD.74.104015. arXiv:hep-th/0607240

9. A. Sheykhi, B. Wang, R.G. Cai, Nucl. Phys. B 779, 1 (2007). https:// doi.org/10.1016/j.nuclphysb.2007.04.028. arXiv:hep-th/0701198

10. M. Akbar, R.G. Cai, Phys. Lett. B 635, 7 (2006). https://doi.org/ 10.1016/j.physletb.2006.02.035. arXiv:hep-th/0602156

11. M. Jamil, E.N. Saridakis, M.R. Setare, Phys. Rev. D 81, 023007 (2010). https://doi.org/10.1103/PhysRevD.81.023007. arXiv:0910.0822 [hep-th]

12. R.G. Cai, N. Ohta, Phys. Rev. D 81, 084061 (2010). https://doi. org/10.1103/PhysRevD.81.084061. arXiv:0910.2307 [hep-th]

13. M. Wang, J. Jing, C. Ding, S. Chen, Phys. Rev. D 81, 083006 (2010). https://doi.org/10.1103/PhysRevD.81.083006. arXiv:0912.4832 [qr-qc] 
14. M. Jamil, E.N. Saridakis, M. Setare, JCAP 11, 032 (2010). https:// doi.org/10.1088/1475-7516/2010/11/032. arXiv:1003.0876 [hepth]

15. Y. Gim, W. Kim, S.H. Yi, JHEP 1407, 002 (2014). https://doi.org/ 10.1007/JHEP07(2014)002. arXiv:1403.4704 [hep-th]

16. Z.Y. Fan, H. Lu, Phys. Rev. D 91(6), 064009 (2015). https://doi. org/10.1103/PhysRevD.91.064009. arXiv:1501.00006 [hep-th]

17. A. Lymperis, E.N. Saridakis, Eur. Phys. J. C 78(12), 993 (2018). https://doi.org/10.1140/epjc/s10052-018-6480-y. arXiv: 1806.04614 [gr-qc]

18. J.D. Bekenstein, Phys. Rev. D 9, 3292-3300 (1974). https://doi. org/10.1103/PhysRevD.9.3292

19. W. Unruh, R.M. Wald, Phys. Rev. D 25, 942-958 (1982). https:// doi.org/10.1103/PhysRevD.25.942

20. J.D. Barrow, Nucl. Phys. B 310, 743-763 (1988). https://doi.org/ 10.1016/0550-3213(88)90101-0

21. B. Wang, E. Abdalla, Phys. Lett. B 471, 346-351 (2000). https:// doi.org/10.1016/S0370-2693(99)01406-9. arXiv:hep-th/9911157

22. M. Setare, JCAP 01, 023 (2007). https://doi.org/10.1088/ 1475-7516/2007/01/023. arXiv:hep-th/0701242

23. I. Fouxon, G. Betschart, J.D. Bekenstein, Phys. Rev. D 77, 024016 (2008). https://doi.org/10.1103/PhysRevD.77.024016. arXiv:0710.1429 [gr-qc]

24. S.F. Wu, B. Wang, G.H. Yang, P.M. Zhang, Class. Quantum Gravity 25, 235018 (2008). https://doi.org/10.1088/0264-9381/25/23/ 235018. arXiv:0801.2688 [hep-th]

25. M. Akbar, Int. J. Theor. Phys. 48, 2665-2671 (2009). https://doi. org/10.1007/s10773-009-0056-4. arXiv:0808.3308 [gr-qc]

26. A. Sheykhi, B. Wang, Phys. Lett. B 678, 434-437 (2009). https://doi.org/10.1016/j.physletb.2009.06.075. arXiv:0811.4478 [hep-th]

27. A. Sheykhi, B. Wang, Mod. Phys. Lett. A 25, 1199-1210 (2010). https://doi.org/10.1142/S0217732310032391. arXiv:0811.4477 [hep-th]

28. N. Mazumder, S. Chakraborty, Gen. Relativ. Gravit. 42, 813-820 (2010). https://doi.org/10.1007/s10714-009-0881-z. arXiv: 1005.3403 [gr-qc]

29. K. Karami, S. Ghaffari, M. Soltanzadeh, Class. Quantum Gravity 27, 205021 (2010). https://doi.org/10.1088/0264-9381/27/20/ 205021. arXiv:1101.3240 [gr-qc]

30. K. Karami, A. Abdolmaleki, JCAP 04, 007 (2012). https://doi.org/ 10.1088/1475-7516/2012/04/007. arXiv:1201.2511 [gr-qc]

31. K. Bamba, M. Jamil, D. Momeni, R. Myrzakulov, Astrophys. Space Sci. 344, 259-267 (2013). https://doi.org/10.1007/ s10509-012-1312-2. arXiv:1202.6114 [physics.gen-ph]

32. K. Karami, T. Najafi, A. Abdolmaleki, Phys. Rev. D 89, 104041 (2014). https://doi.org/10.1103/PhysRevD.89.104041. arXiv:1401.7549 [gr-qc]
33. J.D. Barrow, Phys. Lett. B 808, 135643 (2020). arXiv:2004.09444 [gr-qc]

34. R.K. Kaul, P. Majumdar, Phys. Rev. Lett. 84, 52555257 (2000). https://doi.org/10.1103/PhysRevLett.84.5255. arXiv:gr-qc/0002040

35. S. Carlip, Class. Quantum Gravity 17, 4175-4186 (2000). https:// doi.org/10.1088/0264-9381/17/20/302. arXiv:gr-qc/0005017

36. C. Tsallis, J. Stat. Phys. 52, 479 (1988). https://doi.org/10.1007/ BF01016429

37. G. Wilk, Z. Wlodarczyk, Phys. Rev. Lett. 84, 2770 (2000). https:// doi.org/10.1103/PhysRevLett.84.2770. arXiv:hep-ph/9908459

38. C. Tsallis, L.J.L. Cirto, Eur. Phys. J. C 73, 2487 (2013). https://doi. org/10.1140/epjc/s10052-013-2487-6. arXiv:1202.2154 [condmat.stat-mech]

39. S.A. Hayward, Class. Quantum Gravity 15, 3147-3162 (1998). $\quad$ https://doi.org/10.1088/0264-9381/15/10/017. arXiv:gr-qc/9710089

40. S.A. Hayward, S. Mukohyama, M. Ashworth, Phys. Lett. A 256, 347-350 (1999). https://doi.org/10.1016/ S0375-9601(99)00225-X. arXiv:gr-qc/9810006

41. D. Bak, S.J. Rey, Class. Quantum Gravity 17, L83 (2000). https:// doi.org/10.1088/0264-9381/17/15/101. arXiv:hep-th/9902173

42. B. Wang, Y. Gong, E. Abdalla, Phys. Rev. D 74, 083520 (2006). https://doi.org/10.1103/PhysRevD.74.083520. arXiv:gr-qc/0511051

43. G. Izquierdo, D. Pavon, Phys. Lett. B 633, 420-426 (2006). https:// doi.org/10.1016/j.physletb.2005.12.040. arXiv:astro-ph/0505601

44. H. Mohseni Sadjadi, Phys. Rev. D 73, 063525 (2006). https://doi. org/10.1103/PhysRevD.73.063525. arXiv:gr-qc/0512140

45. H. Mohseni Sadjadi, Phys. Lett. B 645, 108-112 (2007). https:// doi.org/10.1016/j.physletb.2006.12.029. arXiv:gr-qc/0611114

46. M. Szydlowski, A. Stachowski, K. Urbanowski, JCAP 04, 029 (2020). https://doi.org/10.1088/1475-7516/2020/04/029. arXiv: 1812.00616

47. J.P. Mimoso, D. Pavón, Phys. Rev. D 94(10), 103507 (2016). https://doi.org/10.1103/PhysRevD.94.103507. arXiv:1610.07788

48. R.G. Cai, L.M. Cao, Y.P. Hu, JHEP 0808, 090 (2008). https://doi. org/10.1088/1126-6708/2008/08/090. arXiv:0807.1232 [hep-th]

49. N. Radicella, D. Pavon, Phys. Lett. B 691, 121 (2010). https://doi. org/10.1016/j.physletb.2010.06.019. arXiv:1006.3745 [gr-qc]

50. F.K. Anagnostopoulos, S. Basilakos, E.N. Saridakis, Eur. Phys. J. C 80(9), 826 (2020). arXiv:2005.10302 [gr-qc] 